\title{
Therapeutic Capsule Endoscopy: Opportunities and Challenges
}

\author{
Mahdi Rasouli ${ }^{1 *}$, Lin Lin ${ }^{1}$, Andy Prima Kencana1, Kai-Juan Wong², \\ Su-Lim Tan ${ }^{2}$, Khek Yu Ho ${ }^{3}$, Soo Jay Phee ${ }^{1}$ \\ ${ }^{1}$ School of Mechanical and Aerospace Engineering, Nanyang Technological \\ University, Singapore \\ ${ }^{2}$ School of Computer Engineering, Nanyang Technological University, Singapore \\ ${ }^{3}$ National University Hospital, Singapore
}

Submitted December 2010. Accepted for publication July 2011.

\begin{abstract}
The increasing demand for non-invasive (or less-invasive) monitoring and treatment of medical conditions has attracted both physicians and engineers to work together and investigate new methodologies. Wireless capsule endoscopy is a successful example of such techniques which has become an accepted routine for diagnostic inspection of the gastrointestinal tract. This method offers a non-invasive alternative to traditional endoscopy and provides the opportunity for exploring distal areas of the small intestine which are otherwise not accessible. Despite these advantages, wireless capsule endoscopy is still limited in functionality compared to traditional endoscopy. Wireless capsule endoscopes with advanced functionalities, such as biopsy or drug delivery, are highly desirable. In this article, the current status of wireless capsule endoscopy is reviewed together with some of its possible therapeutic applications as well as the existing challenges.
\end{abstract}

Keywords: wireless capsule endoscopy, ingestible medical device, in-body medical device, medical robot, surgical robotics

\section{INTRODUCTION}

Annually, over 60 million people in the US alone are affected by digestive diseases, accounting for about 10 percent of hospitalization and 15 percent of in-patient hospital procedures in the US [1]. Despite advances in medical technology, diagnosis of the cause of the disease remains a mystery in many of these cases. This is mainly due to the

*Corresponding Author: Mahdi Rasouli, School of Mechanical and Aerospace Engineering, Nanyang Technological University, Singapore. Phone: (+65) 6790-5568. Fax: (617) 6793-5921.

E-mail: mahdi@ntu.edu.sg.

Other authors: m070005@e.ntu.edu.sg; andyprima@ntu.edu.sg; askjwong@ntu.edu.sg; assitan@ntu.edu.sg; mdchead@nus.edu.sg; sjphee@ntu.edu.sg. 
limited accessibility of the gastrointestinal (GI) tract, particularly the small intestine, making it difficult to examine the underlying causes non-operatively. Moreover, commonly used investigational techniques addressing the digestive tract are invasive and/or impose a tremendous amount of discomfort to the patient.

Wireless capsule endoscopy, introduced in 2000 by Iddan et al. [2], suggests a noninvasive alternative for inspection of the GI tract. In this method, an electronic pill equipped with a miniature camera is swallowed by the patient. The pill collects images of the digestive tract and wirelessly transmits them to an external device (Figure 1). Extracted images may be viewed in real time [3] or saved into a personal computer [2] for post-processing and analysis. The capsule is then expelled from the body through the natural discharge process.

Wireless capsule endoscopy does not cause discomfort as in the traditional endoscopy, and provides the opportunity for exploring distal areas of the small intestine. This could lead to early diagnosis of GI disorders. However, wireless capsule endoscopy is still limited in functionality compared to traditional endoscopy. Wireless ingestible devices with advanced functionalities such as biopsy and drug delivery, can greatly enhance the diagnosis and treatment of GI disorders and are highly sought after. In this article, we review the current status of capsule endoscopy from a technological point of view, present some of its possible therapeutic applications, and discuss the existing challenges.

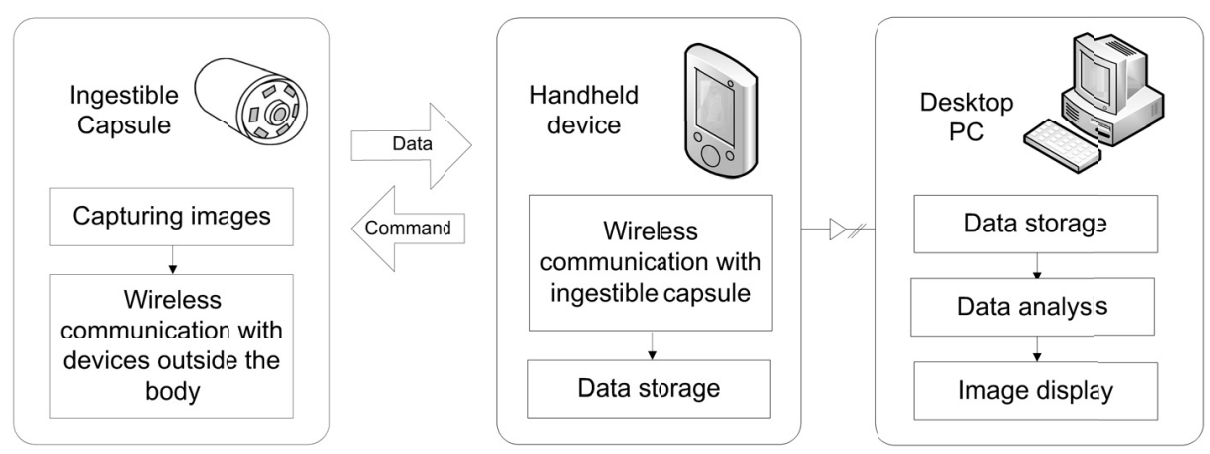

Figure 1. Operational principle of a wireless capsule endoscope.

\section{SYSTEM OVERVIEW}

A conceptual diagram of a wireless capsule endoscope is illustrated in Figure 2. The system includes several core components: an energy source, a processing/control unit, a wireless communication unit, and sensors/actuators. The processing/control unit is the brain of the system and controls the internal operations of the device. Sensors and actuators act as interfaces between the device and body environment. The device can monitor physiological parameters of the body through its sensors and perform therapeutic actions through its actuators. The device communicates with outside the body through a Radio Frequency (RF) link. This enables data exchange between the capsule and the 
external control unit, extraction of the physiological parameters of the body, and control of the device operation. An energy source is needed to power various modules of the device. Details of these components are discussed in the following sections.

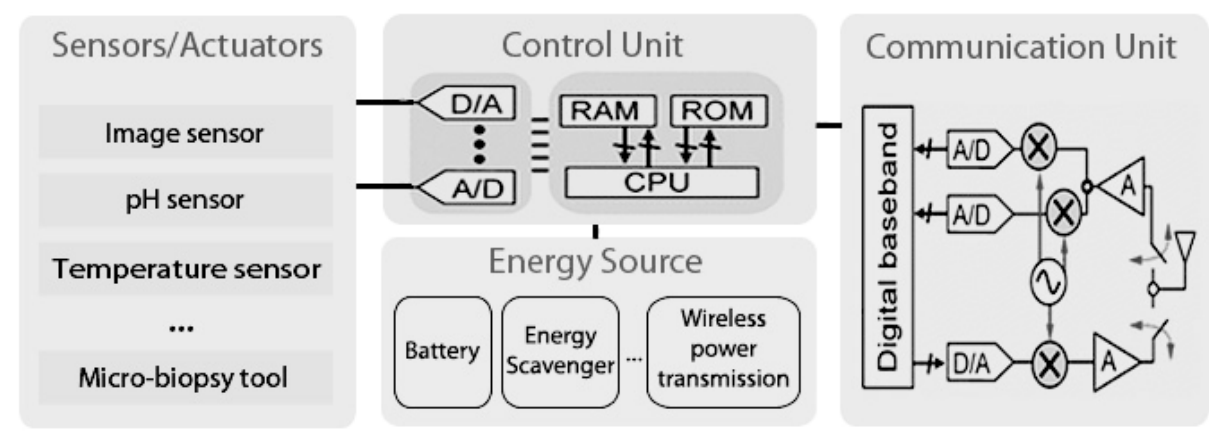

Figure 2. Conceptual schematic of a wireless capsule endoscope.

\subsection{Sensors and Actuators}

Similar to all other medical conditions, monitoring of related physiological parameters is the first step towards the successful diagnosis of GI disorders. Visual inspection of the GI tract and measurement of temperature, $\mathrm{pH}$ value, and pressure are some of the most common methods for detection of GI disorders $[4,5]$. The history of remote monitoring of physiological parameters along the digestive tract dates back to 1957 when the first ingestible capsules for measurement of $\mathrm{pH}$ value were developed [6]. Since then, several wireless ingestible capsules for monitoring of physiological parameters were developed. SmartPill by SmartPill Corporation (New York, USA) [7] is a commercial example of such devices and has gained FDA approval to aid in the evaluation of gastrointestinal disorders. Wireless capsule endoscope introduced by Iddan et al. [2] is another successful example of such devices and has become the 'gold standard' for investigating obscure gastrointestinal bleeding and iron-deficiency anemia [8]. PillCam ${ }^{\mathrm{TM}}$ SB, PillCam ${ }^{\mathrm{TM}}$ ESO, and PillCam ${ }^{\mathrm{TM}}$ COLON by Given Imaging (Yoqneam, Israel) [9], EndoCapsule ${ }^{\mathrm{TM}}$ by Olympus (Tokyo, Japan) [10], MiroCam ${ }^{\mathrm{TM}}$ by IntroMedic (Seoul, Republic of Korea) [11], and OMOM by Chongqing Jinshan Science and Technology Group (Chongqing, China) [12] are some of the wireless capsule endoscopes currently used for inspecting various regions of the GI tract, including small intestine, esophagus, and colon.

Although proven effective for diagnosis of some medical conditions, application of sensors alone for monitoring body parameters provides limited capability and must be usually accompanied by other more-invasive procedures to complete the diagnosis or treatment process. For example, a confirmatory biopsy may be needed after detection of a suspicious lesion through capsule endoscopy. Development of tissue-interactive devices, such as ingestible capsules for drug delivery [13,14] and body tissue/fluid sampling $[15,16]$, is a necessary step towards less invasive solutions for treatment of GI conditions. This is only achievable through integration of actuators in the system. 
Actuators may help performing therapy or improving the diagnosis. In section 3, some of the possible actuation mechanisms for wireless capsule endoscopes are presented.

\subsection{Control Unit}

The control unit controls the operation of various capsule modules and performs desired tasks through proper activation of the sensors and actuators. To this end, an intelligent control unit is embedded in the system through implementation of necessary hardware and software platforms.

The use of "Commercially available Off-The-Shelf" (COTS) components or application-specific integrated circuits (ASICs) technologies are common approaches for the fabrication of the hardware platforms. ASICs provide a dedicated solution with possibly superior performance, smaller size, and lower power consumption, while COTS components offer a less expensive and fast-to-the-market solution for simple applications.

\subsection{Wireless Communication Unit}

Wireless communication with a control unit outside the human body is an essential requirement for wireless capsule endoscopes. This enables data exchange between the capsule and the external control unit, control of the operation of the device, and extraction of physiological parameters of the body.

Large signal attenuation by the body and the stringent size and energy constrains make the development of an efficient wireless communication module for ingestible devices a very challenging task. The wireless communication unit must be small enough so that it can fit into a capsule that could be swallowed by the patient. It must consume low electric power so that it can last long enough to perform useful operations in the digestive tract. Moreover, it should not impose any threat or adverse effect to the human body. For example, generation of heat and electrical current due to transmission of electromagnetic signals should not exceed the regulatory standards [17]. This limits the available range of communication frequency bands for these applications.

Several licensed frequency bands exist specifically for medical applications. The transmission frequency and antenna design are mainly determined by the application. Devices with low bandwidth and data rate can be based on near field transmission (e.g., inductive coupling), while devices with high bandwidth and data rate requirements use radio frequencies. Most of the telemetry-based capsules work in the $402-405 \mathrm{MHz}$ Medical Implant Communication Services (MICS) band [18] or the 433/868/915 MHz Industrial, Scientific and Medical (ISM) band [19], while some employ the $2.4 \mathrm{GHz}$ ISM band [20]. The $2.4 \mathrm{GHz}$ wireless band provides the opportunity of reducing the sizes of antenna and electronic chips and increasing the data rate, at the cost of increased absorption rate and signal attenuation by the body.

Proper choice of communication protocols, modulation techniques, and antenna design are some of the other important factors that can greatly affect the performance of the communication link [21, 22]. Application-specific modulation techniques [23, 24], communication protocols such as medium access control (MAC) protocol [25, 26], and antenna designs $[27,28]$ for ingestible capsules to improve the communication efficiency and reduce the power consumption are reported in the literature. 


\subsection{Energy Source}

Proper functionality of a wireless capsule endoscope relies on a continuous supply of sufficient power. In this sense, a reliable, safe and convenient energy source is imperative.

Currently, wireless capsule endoscopes use electrochemical batteries as their energy source. Electrochemical batteries are relatively large and take almost half of the available space in the capsule. This greatly affects the energy budget of the system and consequently its functionality. Current batteries capacity can sustain capsule's operation for about 8 hours [29]. It is highly desired to extend capsule's lifetime by increasing its energy capacity. Moreover, extending this limitation paves the way for employment of devices with high energy consumptions, such as motors and Shape Memory Alloy (SMA) actuators.

Inductive powering suggests a good alternative for ingestible capsules [30]. However, unpredictable orientation and location of the receiver make the design of a dedicated inductive powering system a very challenging task [31]. Misalignment of transmitting and receiving coils could significantly decrease the transmission efficiency or even result in no received power. To enhance the efficiency and avoid zero crossing in the power output, multiple primary or secondary coils may be employed [31]. Lenaerts et al. [32] developed an inductive power link based on multiple air coils on the receiver side and delivered up to $150 \mathrm{~mW}$ to the capsule. This system was further improved and optimized by Carta et al. [33] with the introduction of a ferrite core. The developed system had the double advantages of reducing the size of the receiving coil set and almost doubling the received power within the same external field.

\section{THERAPEUTIC CAPSULE ENDOSCOPY}

As discussed earlier, current capsule endoscopes suffer from limited functionality. Capsules with advanced functionalities such as active locomotion, body tissue/fluid sampling, and drug delivery can significantly enhance the diagnostic and therapeutic procedures and are highly desirable. In this section, research activities towards therapeutic applications of the wireless capsule endoscopes are reviewed.

\subsection{Active Locomotion}

Commercial ingestible capsules are passive and move by peristalsis. This limits their controllability and thus application for advanced diagnostic and therapeutic purposes. For example, capsule endoscopy may miss the foci of the disease or an area of interest due to fast and unpredictable movements of the capsule. On the other hand, slow movement of the capsule would result in repeated images and waste of the battery energy. Moreover, advanced operations such as body tissue/fluid sampling or hemostasis of bleeding areas are not possible with uncontrolled motion. An active and controllable locomotion could overcome these challenges and open the door for development of systems with advanced functionalities.

Various locomotion mechanisms for ingestible capsules have been proposed. Quirini et al. [34] developed a legged locomotion mechanism using small brushless dc motors (Figure 3). Experimental results demonstrated that the device could travel in the digestive tract with a speed ranging between 10 and $40 \mathrm{~mm} / \mathrm{min}$. The capsule could 
travel a distance of $15 \mathrm{~cm}$ in about 5 minutes against peristalsis in a porcine colon [35].

Kim et al. reported a paddling-based mechanism for active movement of capsule endoscope [36]. Using this mechanism, a mean velocity of $17 \mathrm{~cm} / \mathrm{min}$ over $40 \mathrm{~cm}$ inside the colon of a living pig was achieved.
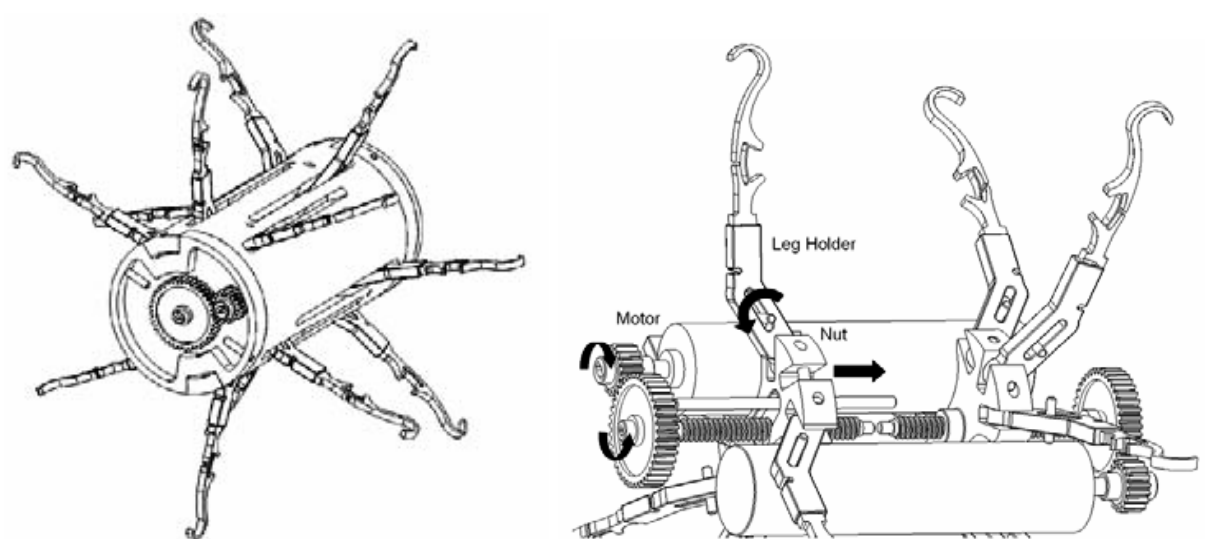

Figure 3. Capsule with legged locomotion [34].

A propeller based solution, actuated by electromagnetic DC motors, was developed by Tortora et al. [37]. It was demonstrated that the propelled capsule could move in all directions in the gastric environment under the control of the endoscopist. A maximum speed of $7 \mathrm{~cm} / \mathrm{s}$ in any directions was achievable using this method. The development was achieved within the frame of the Vector project [38].

Several other methods, such as earthworm-like micro robot [39], vibratory actuation [40], and magnetic guidance [41], have also been investigated. In general, these methods could be classified into two main categories: (1) externally-driven locomotion mechanisms driven by direct force of an external control unit, such as a magnetic generator, and (2) integrated locomotion mechanisms inside the capsule. Externallydriven systems, such as magnetic capsules, are usually simpler in design and can save the space inside the capsule by shifting the complexity towards the external control unit, while integrated locomotion mechanisms are usually more complex, consume more energy, and take more space. Both categories need further research before application in wireless capsule endoscopes.

\subsection{Body tissue/fluid sampling}

Some diagnostic and therapeutic procedures require sampling or even removal of GI tissue or fluids. This is currently performed through either endoscopy or exploratory surgery. A wireless biopsy capsule that can acquire body tissue or fluid at specific locations along the GI tract is highly desirable and has been investigated by several research groups. 
Kong et al. [15] proposed a rotational tissue-cutting razor mechanism. The system is actuated by a torsion spring that is held by a paraffin block at its rotated position. The mechanism is triggered by heating the paraffin block to its melting temperature $\left(40{ }^{\circ} \mathrm{C}\right)$ using an electric coil, releasing the rotational razor. The razor cuts and holds a small piece of the GI mucosa. The sampled tissue could be extracted after the capsule is expelled from the body. The mechanism is housed in an enclosure measuring $10 \mathrm{~mm}$ in diameter and $2 \mathrm{~mm}$ in thickness.

Park et al. [16] developed a microspike biopsy tool. The micro-actuator, illustrated in Figure 4, comprises three parts: a microspike for microbiopsy; an actuating part implementing a slider-crank mechanism using a torsion spring; and a triggering mechanism composed of a SMA heating wire and a polymer string. The microspike is actuated when electric power is applied to the SMA heating wire. The generated heat melts and breaks the string, and consequently releases the torsion spring. This results in generation of a forward and backward movement of the microspike. The fabricated microactuator is $10 \mathrm{~mm}$ in diameter and $1.8 \mathrm{~mm}$ in thickness.

Despite these developments, performing a biopsy in the GI tract using wireless capsules remains a challenge. It is not a trivial task to keep the capsule in close contact with the tissue in order to perform biopsy successfully. This necessitates a controllable locomotion or stopping mechanism that could guide and stop the capsule at a specific location.

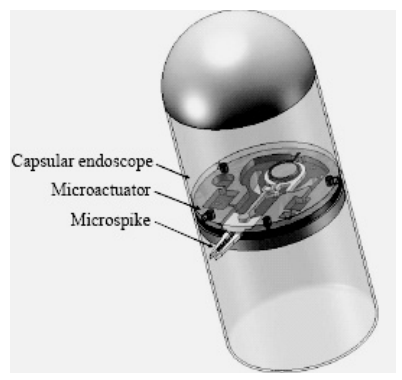

(a)

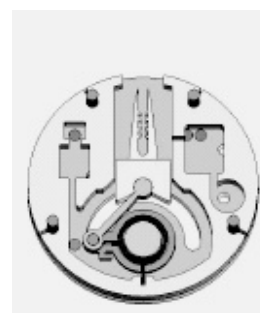

(b)

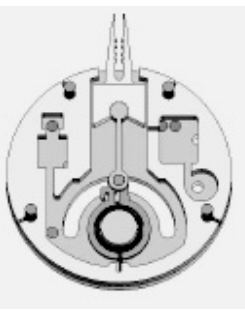

(c)

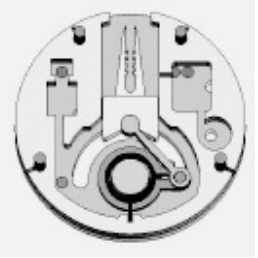

(d)

Figure 4. A capsular endoscope equipped with a micro-actuator for microbiopsy (a), and the micro-actuator in its initial state (b), maximum stroke state (c) and return state (d) [16].

\subsection{Drug Delivery}

Efficient delivery of drugs by ingestible capsule to target organs or body tissues can significantly enhance the efficacy of therapeutic procedures. In the pharmaceutical industry, there has been a significant interest in ingestible capsules that can execute non-invasive drug delivery to selected sites of the human GI tract, in order to determine, for example, drug absorption rate, during the early developmental stages of drugs. 
Enterion capsule [42] by Phaeton Research is a commercial example of drug delivery capsules (Figure 5). It is $11 \mathrm{~mm}$ in diameter, $32 \mathrm{~mm}$ in length, and able to carry $1 \mathrm{ml}$ of drug in either powder or liquid form. Drugs are pushed out of a reservoir by a helicoidal spring. The mechanism is activated by a low frequency magnetic field generator that induces electrical current in a heating element. The generated heat breaks a string that holds the helicoidal spring. This unlocks the spring and triggers the release of the drug from the capsule.

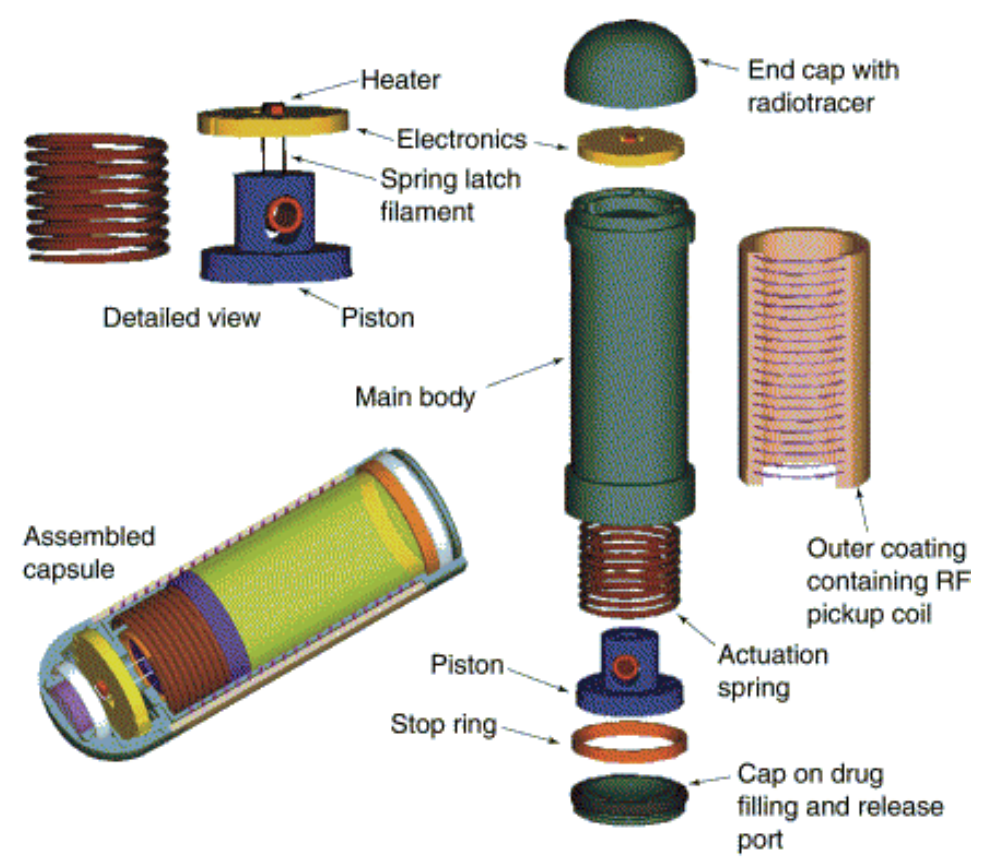

Figure 5. Enterion capsule for drug delivery [42].

Intelisite capsule [13] developed by Innovative Devices is another example of commercial drug delivery systems. The capsule is $10 \mathrm{~mm}$ in diameter, $35 \mathrm{~mm}$ in length, and with a capacity of $0.8 \mathrm{ml}$ of drug solution. The system utilizes SMA wires to generate a rotational motion which creates openings on the capsule and releases the drug.

Several other drug delivery capsules such as controlled release capsule [43], High Frequency (HF) capsule [44], Pulsincap capsule [45] have also been reported. The controlled release capsule employs electrolytic cells to generate gas to push the piston along the capsule. The HF capsule is activated by an RF pulse transmitted from an external generator. The heat generated by the RF current melts a thread and unlocks a needle. The needle pierces a latex balloon prefilled with liquid medication and releases its content. The Pulsincap capsule is equipped with a hydrogel polymer plug which expands upon contact with GI fluids and slowly slides off the capsule body, releasing the stored drug into the GI tract. 


\subsection{Emerging Applications}

Introduction of an intelligent system into the body using a wireless ingestible capsule provides a great opportunity for monitoring and interacting with body environment less invasively. This has lead to the development of new methodologies for diagnosis and treatment of medical conditions.

An ingestible weight management capsule for treatment of obesity is one of such new applications [46]. The operational principle of this technique is illustrated in Figure 6. In this method, a volume-adjustable pill is swallowed by the patient. After the pill enters the stomach, it increases its volume upon receiving a command from an external control unit, and occupies the space in the stomach to induce the feeling of satiety in the patient. The capsule stays in the stomach for a desired period of time. At the end of the treatment period, the pill is deflated and removed from the body through the natural discharge process. The proposed system suggests a good alternative to conventional intragastric balloon (IGB) for treatment of obesity. This approach reduces the discomfort and operational complications caused by the conventional IGBs and provides an opportunity for adjusting the volume of the balloon wirelessly and on demand.

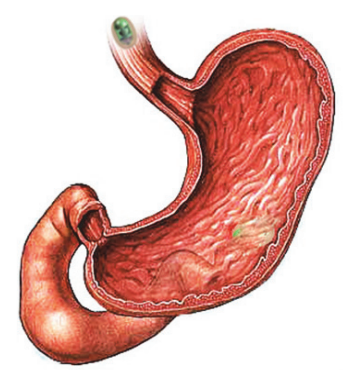

(a) entering the stomach

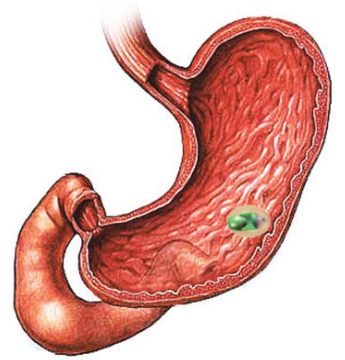

(b) residing in the stomach

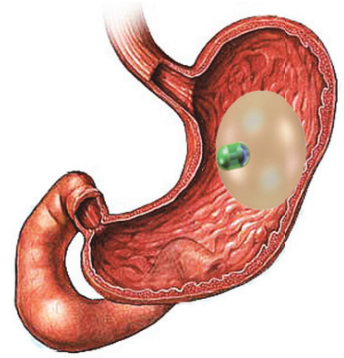

(c) inflation

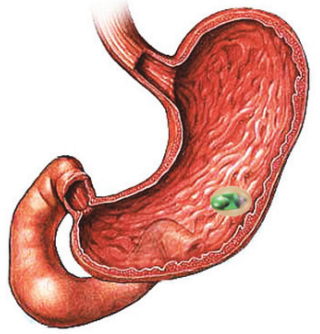

(d) deflation

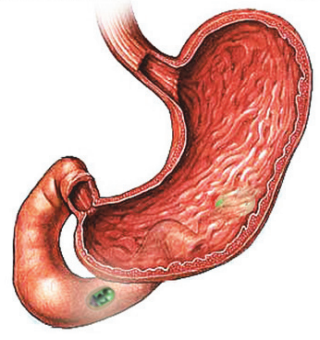

(e) removal (natural discharge)

Figure 6. Operational principle of a capsule for treatment of obesity.

An imaginary futuristic therapeutic capsule endoscope is depicted in Figure 7. A futuristic capsule endoscope may be able to move around and explore desired areas using an active locomotion mechanism and perform advanced diagnostic and therapeutic actions such as tissue sampling or drug delivery. 


\section{TECHNICAL CHALLENGES}

Although current technologies can provide high processing capabilities, the development of more advanced and functional ingestible devices remains a major challenge due to several major constraints, most importantly in limited size and energy budget.

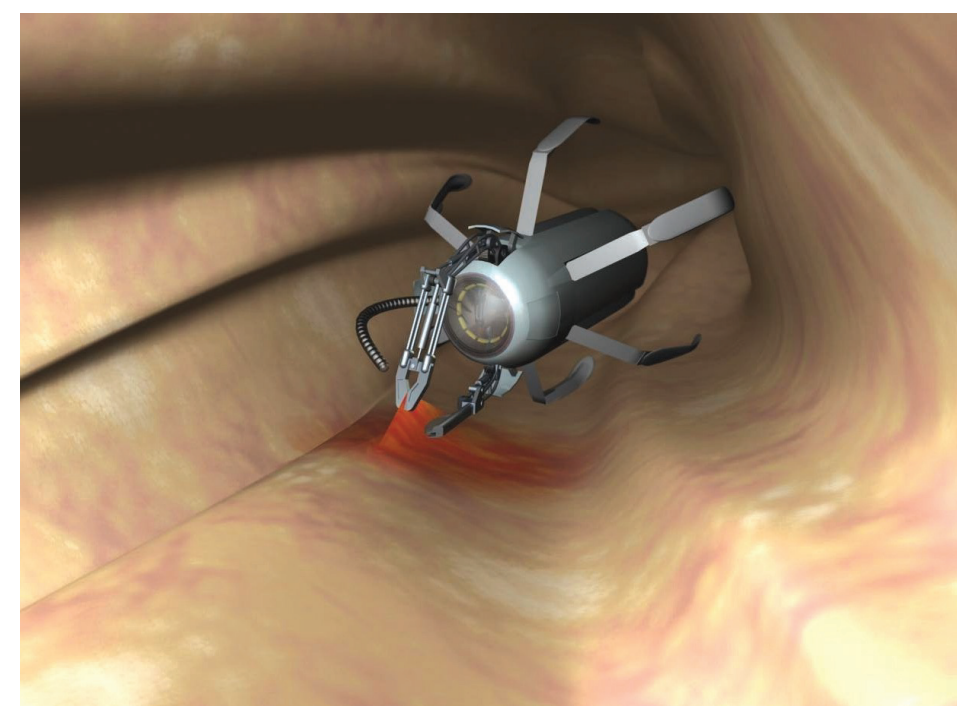

Figure 7. An imaginary depiction of a future wireless capsule endoscope [47].

Miniaturization to obtain an ingestible capsule is a major challenge. The device should be small enough so that it could be swallowed by the patient. Considering dimensions of a typical human digestive system, the capsule must be limited to about 1 $\mathrm{cm}$ in diameter and $3 \mathrm{~cm}$ in length. This imposes a challenging restriction to the system and renders the integration of advanced functional units, particularly mechanical components, impractical due to their relatively large dimensions.

Energy is another major constraint for ingestible medical devices. As discussed earlier, space is very limited in ingestible medical capsules. This includes the available space for on-board energy sources, such as batteries, or energy receivers in the capsule. It is highly desirable to upgrade energy capacity in order to extend the capsule lifetime and to allow integration of functional modules with higher power consumptions such as motors and SMA actuators. On the other hand, the energy issue can be addressed by reducing power consumption of the capsule in operation. This necessitates implementation of energy-efficient, ultra-low power circuit design techniques and effective power management strategies.

Wireless communication through the human body is another major challenge for wireless capsule endoscopy. High signal attenuation and distortion due to the high and 
uneven signal absorption rate of the body poses a great challenge to implementation of a reliable and robust communication link. Moreover, it significantly restricts the usable operational frequency bands and thus the effective data rates. Communication protocols must be energy efficient and implement necessary security and privacy features.

Biocompatibility of the capsule is also a challenge. The capsule should be able to perform its intended functions without eliciting any undesirable local or systemic adverse effects in the body. Moreover, it should be able to survive harsh environmental conditions of the gastrointestinal tract. The capsule is usually encapsulated in a biocompatible material. The encapsulation of contactless sensors, such as image and temperature sensors, is relatively simple compared to the packaging of tissueinteractive actuators or chemical sensors that need direct contact to the GI tissue or fluids.

\section{CONCLUSION}

The demand for non-invasive methodologies for diagnosis and treatment of GI disorders has motivated development of advanced wireless capsule endoscopy systems with diagnostic and therapeutic functionalities. It is envisaged that the wireless capsule endoscopy may most likely replace both diagnostic and interventional flexible endoscopies in the future by providing more functionalities such as body tissue/fluid sampling and drug delivery. The progress towards this goal is mainly focused on the development of miniaturized ultra-low-power functional modules, as well as exploring new methodologies for increasing capsule's energy budget. Recent advances in wireless transmission of data and energy via the human body and the successful manipulation of capsule endoscopes using external magnetic fields have shown promising results for leveraging the functionality of current capsule endoscopy systems. However, these methods need to be further investigated before securing applications in practical systems.

\section{ACKNOWLEDGEMENTS}

This study was funded by Singapore's Agency for Science, Technology and Research (A*STAR) under grant No. 082-140-0036.

\section{CONFLICT OF INTEREST: None}

\section{REFERENCES}

[1] Opportunities and Challenges in Digestive Diseases Research: Recommendations of the National Commission on Digestive Diseases. National Institutes of Health, 2009.

[2] Iddan G, Meron G, Glukhovsky A, Swain P. Wireless capsule endoscopy. Nature, 2000, 405: 417-417.

[3] Ogata H, Kumai K, Imaeda H, Aiura K, Hisamatsu T, Okamoto S, Iwao Y, Sugino Y, Kitajima M, Hibi T. Clinical Impact of a Newly-Developed Capsule Endoscope: Usefulness of a Real Time Image Viewer Against Gastrointestinal Transit Abnormality. Gastrointestinal Endoscopy, 2006, 63(5): AB177-AB177.

[4] Wingate D, Hongo M, Kellow J, Lindberg G, Smout A. Disorders of gastrointestinal motility: Towards a new classification. Journal of Gastroenterology and Hepatology, 2002, 17: S1-S14.

[5] Sleisenger MH, Fordtran JS. Gastrointestinal disease: pathophysiology, diagnosis, management, 5th ed., W. B. Saunders, Philadelphia, 1993. 
[6] Mackay RS, Jacobson B. Endoradiosonde. Nature, 1957, 179: 1239-1240.

[7] http://www.smartpillcorp.com/, last accessed: 1 Nov 2010.

[8] Moglia A, Menciassi A, Dario P, Cuschieri A. Capsule endoscopy: progress update and challenges ahead. Nat Rev Gastroenterol Hepatol, 2009, 6(6): 353-361.

[9] http://www.givenimaging.com/, last accessed: 15 June 2010.

[10] http://www.olympusamerica.com/msg_section/index.asp, last accessed: 15 May 2010.

[11] http://www.intromedic.com/en/product/productInfo.asp, last accessed: 1 April 2010.

[12] http://www.jinshangroup.com, last accessed: 15 June 2010.

[13] Parr AF, Sandefer EP, Wissel P, McCartney M, McClain C, Ryo UY, Digenis GA. Evaluation of the Feasibility and Use of a Prototype Remote Drug Delivery Capsule (RDDC) for Non-Invasive Regional Drug Absorption Studies in the GI Tract of Man and Beagle Dog. Pharmaceutical Research, 1999, 16(2): 266-271.

[14] Wilding I, Hirst P, Connor A. Development of a new engineering-based capsule for human drug absorption studies. Pharmaceutical Science \& Technology Today, 2000, 3(11), 385-392.

[15] Kong KC, Cha J, Jeon D, Cho DD. A rotational micro biopsy device for the capsule endoscope. Conf Proc IEEE/RSJ International Conference on Intelligent Robots and Systems. 2005, 1839 - 1843

[16] Park S, Koo K, Bang SM, Park JY, Song SI, Cho DD. A novel microactuator for microbiopsy in capsular endoscopes. Journal of Micromechanics and Microengineering, 2008, 18(2): 25-32.

[17] IEEE standard for safety levels with respect to human exposure to radio frequency electromagnetic fields, $3 \mathrm{kHz}$ to $300 \mathrm{GHz}$. IEEE Std C951, 2006.

[18] Bradley PD, An ultra low power, high performance Medical Implant Communication System (MICS) transceiver for implantable devices. 2006, Conf Proc IEEE Biomedical Circuits and Systems, 158-161.

[19] Chen X, Zhang X, Zhang L, Li X, Qi N, Jiang H, Wang Z. A wireless capsule endoscope system with low-power controlling and processing ASIC. IEEE Transactions on Biomedical Circuits and Systems, 2009, 3(1): 11-22.

[20] Chi B, Yao J, Han S, Xie X, Li G, Wang Z. A 2.4 GHz low power wireless transceiver analog front-end for endoscopy capsule system. Analog Integrated Circuits and Signal Processing, 2007, 51(2): 59-71.

[21] Bhargava A, Zoltowski M, Sensors and wireless communication for medical care. Conf Proc International Workshop on Database and Expert Systems Applications. 2003, 1: 956-960.

[22] Yang H, Robert F. Wireless body sensor networks for health-monitoring applications. Physiological Measurement, 2008, 29(11): R27-R56.

[23] Kihyun K, Sungho L, Eunil C, Junghee C, Sangwook N, Design of OOK system for wireless capsule endoscopy. Conf Proc IEEE International Symposium on Circuits and Systems. 2010, 1205-1208.

[24] Jung-Yeol O, Jae-Hwan K, Hyung-Soo L, Jae-Young K. PSSK Modulation Scheme for High-Data Rate Implantable Medical Devices. IEEE Transactions on Information Technology in Biomedicine, 2010, 14(3): 634-640.

[25] Aydin N, Arslan T. Review of Communication Systems for Ingestible Miniaturized Integrated Sensor Microsystems. Advanced Technologies for Enhanced Quality of Life. Iasi, Romania, 2009, 91-95.

[26] Lin L, Wong KJ, Tan SL, Phee SJ. Asymmetric Multihop Networks for Multi-Capsule Communications within the Gastrointestinal Tract. Body Sensor Network. CA, USA, 2009.

[27] Izdebski PM, Rajagopalan H, Rahmat-Samii Y. Conformal ingestible capsule antenna: A novel chandelier meandered design. IEEE Transactions on Antennas and Propagation, 2009, 57(4): 900-909.

[28] Kwak S, Chang K, Yoon YJ. Small spiral antenna for wideband capsule endoscope system. Electronics Letters, 2006, 42(23): 1328-1329.

[29] Swain P. The future of wireless capsule endoscopy. World Journal of Gastroenterology, 2008, 14(26): 4142-4145.

[30] Rasouli M, Phee L. Energy sources and their development for application in medical devices. Expert review of medical devices, 2010, 7(5): 693-709. 
[31] Guanying M, Guozheng Y, Xiu H. Power transmission for gastrointestinal microsystems using inductive coupling. Physiological Measurement, 2007, 28(3): 9-18.

[32] Lenaerts B, Puers R. Omnidirectional Inductive Powering for Biomedical Implants, Springer Netherlands, 2009.

[33] Carta R, Thoné J, Puers R. A wireless power supply system for robotic capsular endoscopes. Sensors and Actuators A: Physical, 2010, 162(2): 177-183.

[34] Quirini M, Menciassi A, Scapellato S. Design and fabrication of a motor legged capsule for the active exploration of the gastrointestinal tract. IEEE Trans Mechatronics, 2008, 13: 169-179.

[35] Marco Q, Arianna M, Sergio S, Paolo D, Fabian R, Chi-Nghia H, Sebastian S, Marc Oliver S. Feasibility proof of a legged locomotion capsule for the GI tract. Gastrointestinal Endoscopy, 2008, 67(7): 1153-1158.

[36] Kim HM, Yang S, Kim J, Park S, Cho JH, Park JY, Kim TS, Yoon E-S, Song SY, Bang S. Active locomotion of a paddling-based capsule endoscope in an in vitro and in vivo experiment. Gastrointestinal Endoscopy, 2010, 72(2): 381-387.

[37] Tortora G, Valdastri P, Susilo E, Menciassi A, Dario P, Rieber F, Schurr MO. Propeller-based wireless device for active capsular endoscopy in the gastric district. Minimally Invasive Therapy and Allied Technologies, 2009, 18(5): 280-290.

[38] http://www.vector-project.com, last accessed: 15 June 2010.

[39] Kim B, Lee MG, Lee YP, Kim Y, Lee G. An earthworm-like micro robot using shape memory alloy actuator. Sensors and Actuators A: Physical, 2006, 125(2): 429-437.

[40] Carta R, Pateromichelakis N, Thoné J, Sfakiotakis M, Tsakiris DP, Puers R. A wireless powering system for a vibratory-actuated endoscopic capsule. Procedia Engineering, 2010, 5: 572-575.

[41] Swain P, Toor A, Volke F, Keller J, Gerber J, Rabinovitz E, Rothstein RI. Remote magnetic manipulation of a wireless capsule endoscope in the esophagus and stomach of humans. Gastrointestinal Endoscopy, 2010, 71(7): 1290-1293.

[42] Wilding I, Hirst P, Connor A. Development of a new engineering-based capsule for human drug absorption studies. Pharmaceutical Science and Technology Today, 2000, 3(11): 385-392.

[43] Gröning R, Bensmann H, Müller RS. Control of drug release from capsules using high frequency energy transmission systems. International Journal of Pharmaceutics, 2008, 364(1): 9-13.

[44] Fuhr U, Staib AH, Harder S, Becker K, Liermann D, Schollnhammer G, Roed IS. Absorption of ipsapirone along the human gastrointestinal tract. Br J Clin Pharmacol, 1994, 38(1): 83-86.

[45] Stevens HNE, Wilson CG, Welling PG, Bakhshaee M, Binns JS, Perkins AC, Frier M, Blackshaw EP, Frame MW, Nichols DJ, Humphrey MJ, Wicks SR. Evaluation of Pulsincap(TM) to provide regional delivery of dofetilide to the human GI tract. International Journal of Pharmaceutics, 2002, 236(1-2): 27-34.

[46] Kencana AP, Rasouli M, Huynh VA, Ting EK, Chong YL, Nguyen DQH, Tan SL, Wong KJ, Phee SJ. An Ingestible Wireless Capsule for Treatment of Obesity. 32nd Annual Conference of IEEE Engineering in Medicine and Biology Society. Minneapolis, Argentina, 2010.

[47] http://www.sintef.no/home/Technology-and-Society/Medical-technology/Ultrasound-and-imageguided-therapy/Laparoscopic-surgery/Vector, last accessed: 1 June 2011 


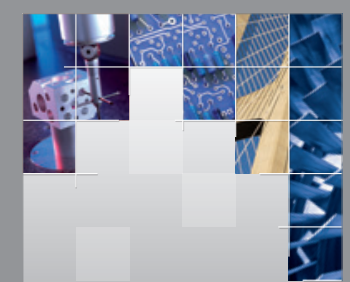

\section{Enfincering}
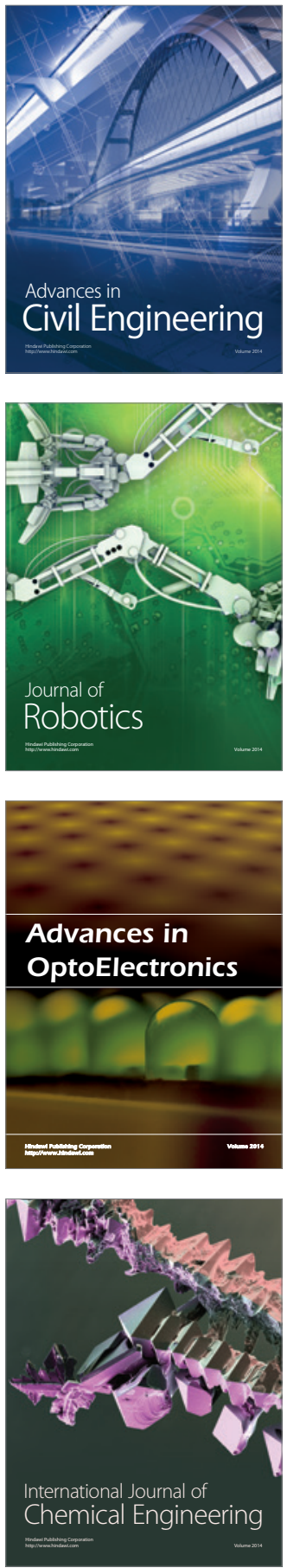

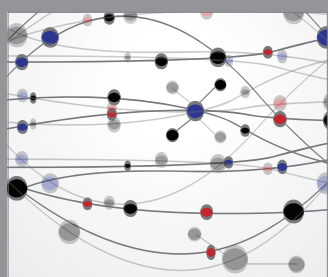

The Scientific World Journal

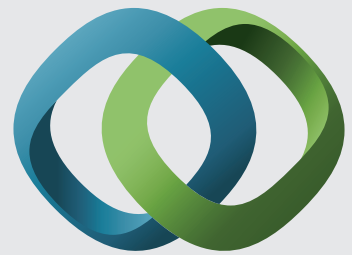

\section{Hindawi}

Submit your manuscripts at

http://www.hindawi.com
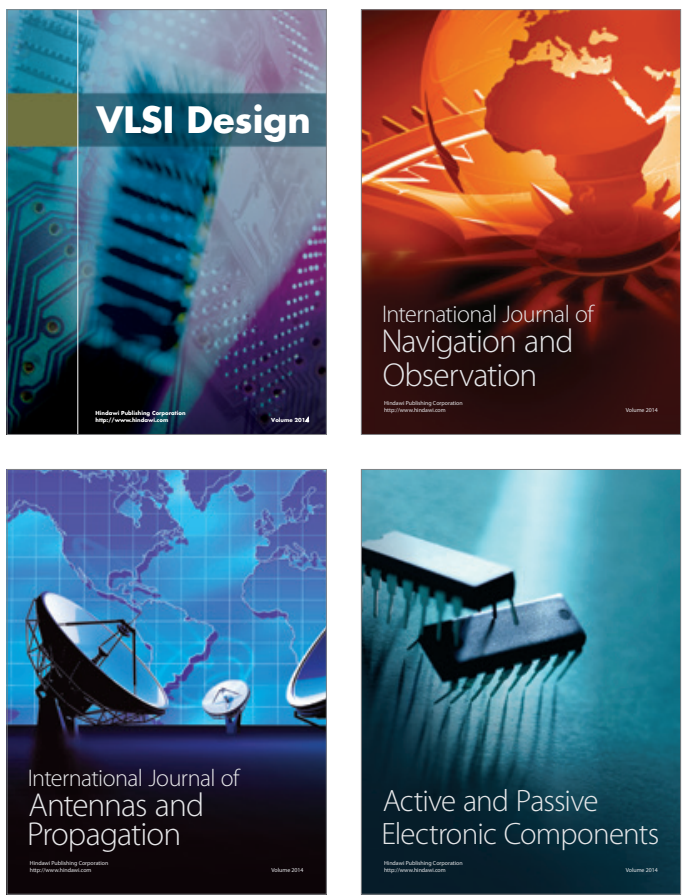
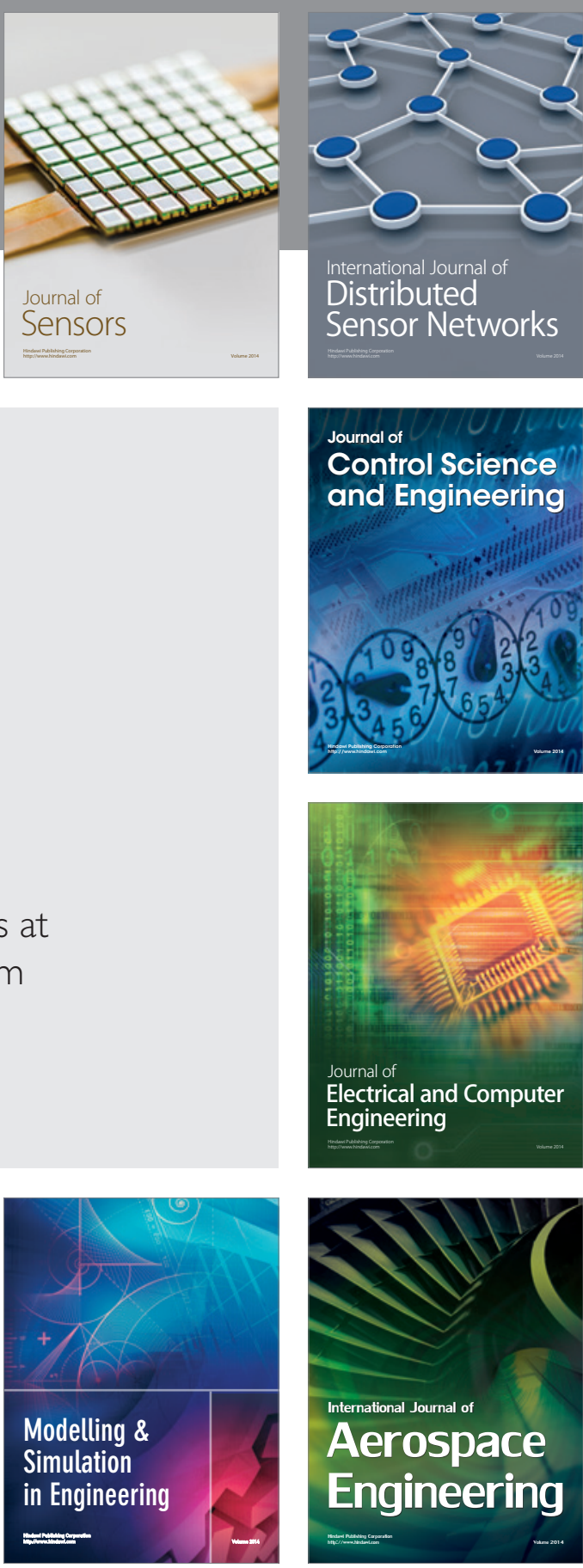

International Journal of

Distributed

Sensor Networks

Journal of

Control Science

and Engineering
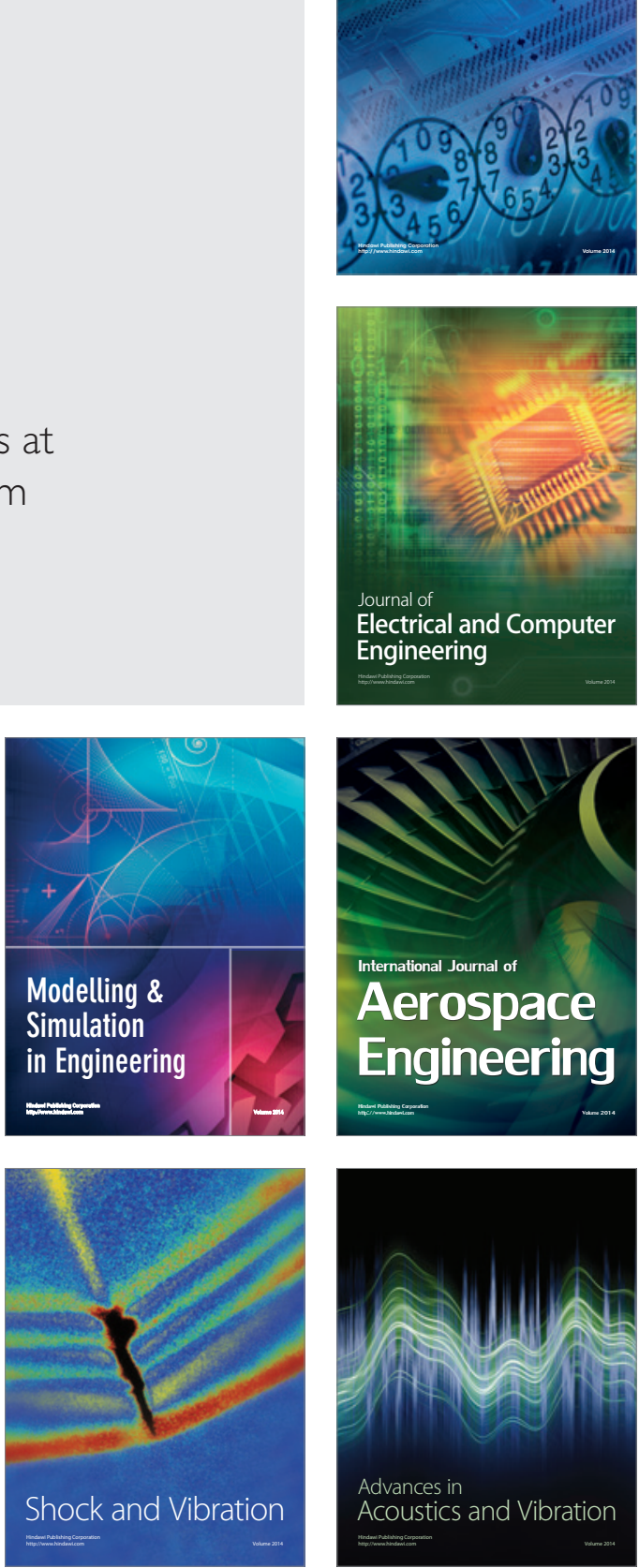